\title{
Sensorimotor Cortex Activation during Mirror Therapy in Healthy Right-Handed Subjects: A Study with Near-Infrared Spectroscopy
}

\author{
ITSUKI IMAI ${ }^{1,2)^{*}}$, KOTARO TAKEDA ${ }^{2,3)^{*}}$, TAIZO ShIOMI ${ }^{2,4)}$, TAKAMICHI TANIGUCHI ${ }^{5)}$, \\ HIROYUKI KATO ${ }^{2,3)}$ \\ 1) Department of Physical Therapy, Nasu Neurosurgical Center \\ 2) CREST, Japan Science and Technology Agency \\ ${ }^{3)}$ Department of Neurology, International University of Health and Welfare Hospital: 537-3 \\ Iguchi, Nasushiobara, Tochigi 329-2763, Japan. \\ TEL +81 287-39-3060, FAX +81 287-39-3001, E-mail: katoh@iuhw.ac.jp \\ 4) Department of Physical Therapy, International University of Health and Welfare \\ ${ }^{5)}$ Department of Occupational Therapy, International University of Health and Welfare \\ *: Equal contribution
}

J. Phys. Ther. Sci.

20: $141-145,2008$

\begin{abstract}
Clinical studies have shown that functional motor recovery after stroke may be facilitated by mirror therapy (MT). However, its underlying mechanism is uncertain. In this study, we examined brain activation during unilateral hand movement in 5 right-handed healthy subjects ( 1 male and 4 females) with or without viewing a mirror reflection of the moving hand (MT). We measured the changes in the concentration of oxygenated hemoglobin in the primary sensorimotor cortex (SMC) using near-infrared spectroscopy. We calculated the laterality index (LI) using the extent of activation in the right and left SMCs. The LIs ( \pm standard error of mean) during right hand grasping without and with MT were $0.17 \pm$ 0.11 (left SMC predominance) and $-0.42 \pm 0.24$ (right SMC predominance), respectively $(\mathrm{p}<0.05$ ). However, there was no significant difference in LIs during left hand grasping without and with MT $(0.02 \pm$ 0.05 and $0.08 \pm 0.05$, respectively). The findings suggest that MT is more effective when it is used for dominant right hand movement, and this phenomenon may be the related to the different manipulability between the dominant and non-dominant hands.
\end{abstract}

Key words: NIRS, Laterality, Mirror therapy

(This article was submitted Jan. 7, 2008, and was accepted Mar. 3, 2008)

\section{INTRODUCTION}

Nearly ten years ago, mirror therapy (MT) was introduced as a useful technique for the prevention and treatment of phantom pain ${ }^{1)}$. The mirror was placed vertically in front of an amputee, who moved the non-injured hand while watching its reflection, which was visually superimposed on the amputated hand (phantom limb). Despite the fact that the patients with phantom limb could not move the amputated hand, the reflection of the intact hand movement led to perceptions of movement in the phantom limb. Subsequently, MT has also been used to improve the function of the paretic hand in patients with stroke ${ }^{2)}$. At present, MT is known as a rehabilitation approach for functional motor recovery of an impaired $\operatorname{limb}^{3,4)}$ and for various pain reductions $^{5-7)}$.

Clinical studies have shown that functional motor recovery after stroke may be facilitated by 
$\mathrm{MT}^{2,8-10)}$. However, few studies have measured brain activation during MT. In other words, little attempt has been made to give a functional explanation for the relation between MT effects and cerebral cortical activation.

Near-infrared spectroscopy (NIRS), which can monitor the changes in concentrations of oxygenated and deoxygenated hemoglobin $(\Delta[\mathrm{Oxy}-$ $\mathrm{Hb}]$ and $\Delta[$ Deoxy-Hb], respectively), provides noninvasive visualization of neural activity in the brain ${ }^{11)}$. An advantage of NIRS is the unnecessity of restraint because of resistance to motion artifacts when the NIRS probes are mounted securely ${ }^{12)}$. Therefore, the subjects could perform the hand grasping task with mirror viewing during NIRS measurement with a natural posture without any constraint. The purpose of this study was to investigate cerebral activation, mainly in the sensorimotor cortex (SMC), during MT using NIRS from the viewpoint of cerebral laterality.

\section{MATERIALS AND METHODS}

The subjects were 5 right-handed healthy volunteers, a male and four females $(21.2 \pm 1.1$ years of age). Experiments were performed after obtaining informed consent from each subject according to the Declaration of Helsinki.

Subjects were seated with their hands in the neutral position (forearm pronation/supination $0^{\circ}$ ) on a table. The motor task involved a simple unilateral hand grasping movement. One complete grasping cycle (open-close of the hand) was performed in synchrony to auditory metronome beats ( 60 beats/minute). Each 20 -s motor task period ( 3 times) was alternated by a 40 -s rest period. The motor task was performed under the following four conditions (Fig. 1). A: right hand grasping while viewing the inactive left hand. B: right hand grasping while viewing a mirror reflecting the active right hand. C: left hand grasping while viewing the inactive right hand. D: left hand grasping while viewing a mirror reflecting the active left hand. In the inactive hand viewing conditions, subjects watched the inactive hand while moving the other hand. In the mirror viewing conditions (MT), subjects watched a mirror reflection of the active hand, with the inactive hand positioned unseen behind the mirror.

The NIRS used in this study was an optical topography system (ETG-4000, Hitachi Medical Co.,
A

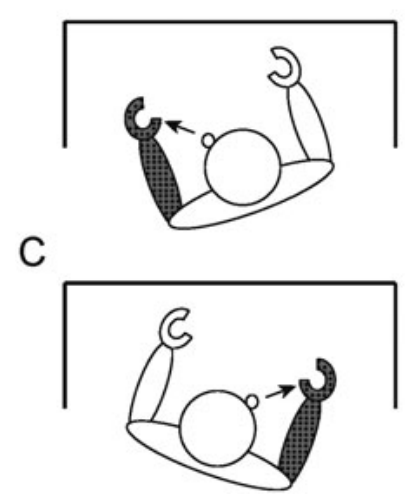

B

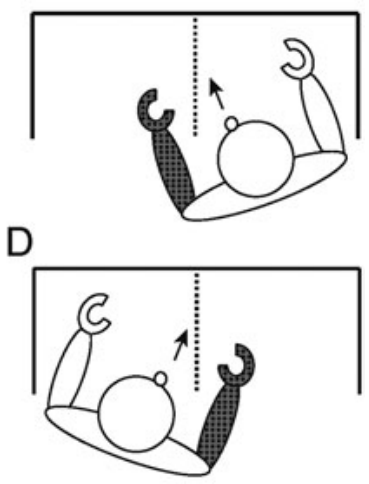

Fig. 1. The four experimental task conditions. A: Right hand grasping while viewing the inactive left hand. $\mathrm{B}$ : Right hand grasping while viewing a mirror that reflects the active right hand. C: Left hand grasping while viewing the inactive right hand. D: Left hand grasping while viewing a mirror that reflects the active left hand. Arrow: direction of the gaze. Dotted line: mirror. Grey hand: inactive hand.

Tokyo, Japan). A couple of head shells mounting 5 light-emission probes and 4 light-detection probes covered the scalp overlying both SMCs. Because C3 and $\mathrm{C} 4$ points of the international 10-20 system for electroencephalography are thought to be located on the pre- and post-central gyri $\left.{ }^{13}, 14\right)$ referred to as $\mathrm{SMC}^{15)}$, we defined a set of 7 channels of both sides on and around C3 and C4 (Fig. 2) as SMC regions of interest (ROI).

The wavelengths of the two kinds of laser diodes were 695 and $830 \mathrm{~nm}$, and the distance between the nearest light-emission and light-detection probes was $30 \mathrm{~mm}$. The scalp was illuminated with the two near-infrared laser beams and reflectance beams sampled at $10 \mathrm{~Hz}$ were used to calculate $\Delta[\mathrm{Oxy}-\mathrm{Hb}]$ and $\Delta[$ Deoxy-Hb]. The linear trends of the continuous $\Delta[\mathrm{Oxy}-\mathrm{Hb}]$ and $\Delta[\mathrm{Deoxy}-\mathrm{Hb}]$ fluctuations were removed. The changes in the $\mathrm{Hb}$ concentrations were smoothed with a 5-s moving average, and were averaged synchronously to the target grasping blocks after excluding the blocks with artifacts. Experimental data of $\Delta[\mathrm{Oxy}-\mathrm{Hb}]$ was focused because $\Delta[\mathrm{Oxy}-\mathrm{Hb}]$ is the most sensitive marker of activation-dependent changes in regional cerebral blood flow ${ }^{15)}$. The standard deviation (SD) was calculated against 5-s rest periods just before the target grasping blocks and then each set of data of $\Delta[\mathrm{Oxy}-\mathrm{Hb}]$ was transformed into a $\mathrm{z}$ score through division by the SD of each 


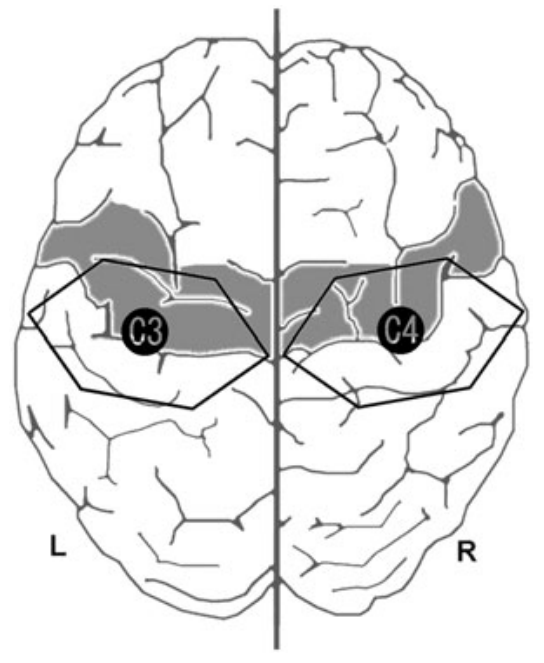

Fig. 2. The hexagons show the positions of the shells mounting the NIRS probes (region of interest). The grey cortical areas denote the primary motor cortex. $\mathrm{C} 3$ and $\mathrm{C} 4$ points of the 10-20 system correspond to the pre- and post-central gyri.

channel. The response of each channel in the ROI was deemed significant when $\Delta[\mathrm{Oxy}-\mathrm{Hb}]$ was above the significance level (z score $>3$, for multiple comparison) during the target grasping block. Because NIRS data are semiquantitative, we did not use the amplitude of $\Delta[\mathrm{Oxy}-\mathrm{Hb}]$ in each NIRS channel as a marker of activation, but used the number of channels that showed a significant increase in $\Delta[\mathrm{Oxy}-\mathrm{Hb}]^{16)}$. To determine which cerebral hemisphere was activated predominantly in the experimental paradigm, we used a laterality index $(\mathrm{LI})$ calculated as $\mathrm{LI}=(\mathrm{ML}-\mathrm{MR}) /(\mathrm{ML}+\mathrm{MR})$, where ML and MR were the activation areas during the grasping task in the ROI of the left and right SMCs, respectively. The LI ranges from -1 (exclusively right hemispheric predominance) to +1 (exclusively left hemispheric predominance) ${ }^{16)}$. The extent of activation in each hemisphere and the LIs of all the experimental conditions were compared with one-way ANOVA. These analyses were performed using MATLAB 7.0 (Mathworks, Natick, MA, USA).

\section{RESULTS}

Task-related transient increases in $\Delta[\mathrm{Oxy}-\mathrm{Hb}]$ and slight decreases in $\Delta[$ Deoxy-Hb] were observed in all the channels that were deemed significantly activated. An example of $\Delta[\mathrm{Hb}]$ measured under

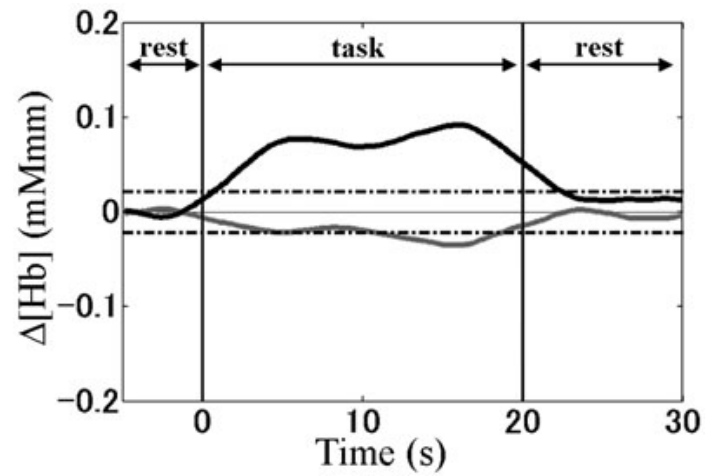

Fig. 3. An example of $\Delta[\mathrm{Hb}]$ at the channel over the $\mathrm{C} 3$ point during right hand grasping under the inactive hand viewing condition. Black thick line: $\Delta[$ Oxy$\mathrm{Hb}]$; Grey line: $\Delta[$ Deoxy-Hb]; Vertical line: task onset and offset timing; Dash-dotted line: significance levels $( \pm 3 \times \mathrm{SD})$ of $\Delta[\mathrm{Oxy}-\mathrm{Hb}]$.

condition $\mathrm{A}$ is shown in Fig. 3, and was a typical activation pattern.

During mirror viewing conditions (conditions B and D), all the subjects experienced an odd feeling known as motor illusion.

There were no significant differences in the extent of activation in the right and left hemispheres under all the conditions $(\mathrm{F}(7,32)=0.92, \mathrm{p}=0.505)$. However, there was a significant difference among conditions with regard to laterality $(\mathrm{F}(3,16)=3.67$, $p<0.05$ ). The LIs (mean \pm standard error of mean) of all the conditions are shown in Table 1 and Fig. 4. Brain activation during right hand grasping under the inactive hand watching condition (condition $\mathrm{A}$ ) demonstrated a slight, left hemispheric predominance $(\mathrm{LI}=0.17 \pm 0.11)$. The LI of condition A was significantly larger than that of right hand grasping under the mirror viewing condition (condition $\mathrm{B}, \mathrm{LI}=-0.42 \pm 0.24$ showing right hemispheric predominance) $(\mathrm{F}(3,16)=3.67, \mathrm{p}$ $=0.035$, posthoc Tukey's HSD test). In contrast, left hand grasping tasks (conditions $\mathrm{C}$ and $\mathrm{D}$ ) showed unclear laterality of activation with or without mirror viewing ( $\mathrm{LI}=0.02 \pm 0.05,0.08 \pm$ 0.05 , respectively) and there was no significant difference between them.

\section{DISCUSSION}

The present NIRS study investigated the effect of MT on SMC activation during unilateral hand movement. During right hand grasping without 


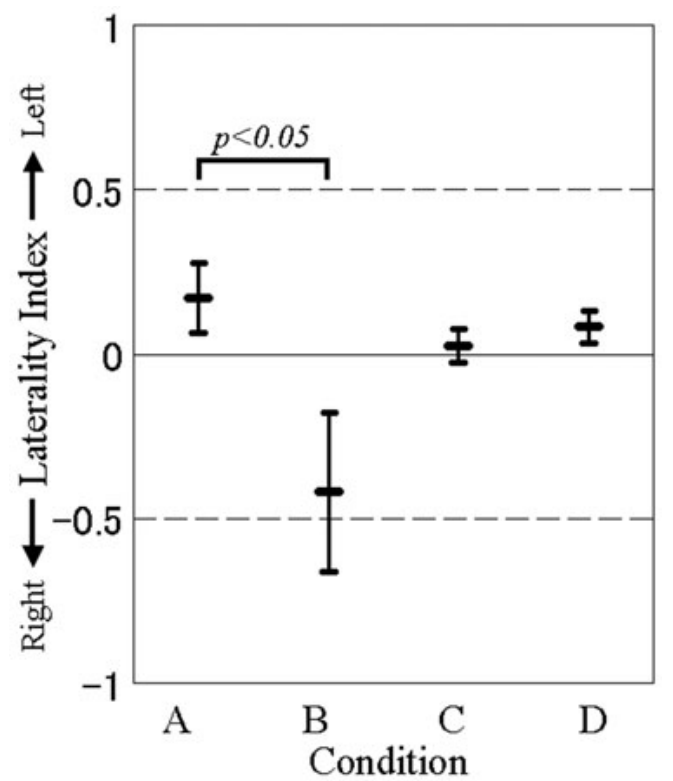

Fig. 4. The laterality index (LI) under each experimental condition. There was a significant difference in LI only between conditions A and B (right hand grasping without and with viewing the mirror, respectively).

Table 1. Laterality Indices (mean \pm standard error of mean)

\begin{tabular}{crl}
\hline Condition & \multicolumn{1}{c}{ L1 } & pvalue \\
\hline A & $0.17 \pm 0.11$ & $0.035^{*}$ \\
B & $-0.42 \pm 0.24$ & \\
\hline C & $0.02 \pm 0.05$ & 0.991 \\
D & $0.08 \pm 0.05$ & \\
\hline
\end{tabular}

mirror viewing (condition A), the LI showed a contralateral SMC predominant activation pattern. This activation pattern was the same as that observed in previous NIRS studies ${ }^{11,12,16)}$. However, the same subjects exhibited LI that was close to zero during left hand grasping without mirror viewing (condition $\mathrm{C}$ ). This phenomenon is in accordance with an earlier fMRI study that reported bilateral SMC activation when healthy subjects moved their non-dominant hand ${ }^{17)}$.

Interestingly, the SMC activation during condition $\mathrm{B}$ (right hand grasping while viewing a mirror) demonstrated a significant decrease in LI (toward right hemispheric predominance) compared to that during condition A, although the area of SMC activation or the number of activated channels in each hemisphere was similar among all the conditions (A to D). However, there was no shift of activation by MT during left hand grasping. The present findings suggest that the change of laterality balance by MT is specific to dominant hand movement. Although it is difficult to compare the quality and the amount of motor illusion (odd feeling) during mirror viewing, we hypothesize that motor illusion occurs more easily when the dominant hand is moving, and the non-dominant hand is hidden behind the mirror reflection of the moving hand, than when the non-dominant hand is moving, and the dominant hand is hidden behind the reflection of moving hand, probably due to the difference in manipulability between the dominant and non-dominant hands. If our hypothesis is correct, stroke or amputee patients might experience motor illusion more easily in MT than healthy subjects.

The present result of SMC activation during MT is different from the result of an earlier study ${ }^{18)}$, which described no difference in ipsilateral M1 excitability between the dominant and nondominant hands. The reason for this difference is unclear at present, but a difference in methodology, NIRS versus transcranial magnetic stimulation (TMS), may be the cause because the NIRS measures $\mathrm{Oxy}-\mathrm{Hb}$ changes, an indirect measure of brain activity, while TMS explores brain activity via direct stimulation of motor cortical areas.

The present study showed that the brain activation pattern during hand movement was modified by MT. The result suggests that MT has an effect on cerebral activities that potentially contribute to functional motor recovery of an impaired $\operatorname{limb}^{2-4}$ ). The mechanism underlying the recovery of motor function after stroke is known to be a use-dependent neural plasticity ${ }^{19)}$. The cortical plasticity has been demonstrated in terms of expansion of topographic maps by various therapeutic interventions, such as constraint-induced movement therapy ${ }^{20)}$ and motor imagery $^{21)}$. Although the mechanism is not clear enough, changes in cerebral activation induced by MT may play an important role in inducing neural plasticity. Previous functional imaging studies have suggested that several motor related areas (premotor cortex, supplementary motor areas, and cerebellum) as well as SMC contribute to motor recovery after stroke $^{22-25)}$. Therefore, it would be interesting to consider the effect of MT on these motor related areas in future studies. 


\section{ACKNOWLEDGEMENT}

This study was supported by CREST, Japan Science and Technology Agency.

\section{REFERENCES}

1) Ramachandran VS, Rogers-Ramachandran D, Cobb S: Touching the phantom limb. Nature, 1995, 377: 489490.

2) Altschuler EL, Wisdom SB, Stone L, et al.: Rehabilitation of hemiparesis after stroke with a mirror. Lancet, 1999, 353: 2035-2036.

3) Byl NN, McKenzie A: Treatment effectiveness for patients with a history of repetitive hand use and focal hand dystonia: a planned, prospective follow-up study. J Hand Ther, 2000, 13: 289-301.

4) Rosen B, Lundborg G: Training with a mirror in rehabilitation of the hand. Scand J Plast Reconstr Surg Hand Surg, 2005, 39: 104-108.

5) Giraux P, Sirigu A: Illusory movements of the paralyzed limb restore motor cortex activity. Neuroimage, 2003, 20(Suppl 1): S107-111.

6) McCabe CS, Haigh RC, Ring EF, et al.: A controlled pilot study of the utility of mirror visual feedback in the treatment of complex regional pain syndrome (type 1). Rheumatology (Oxford), 2003, 42: 97-101.

7) Moseley GL: Graded motor imagery is effective for long-standing complex regional pain syndrome: a randomised controlled trial. Pain, 2004, 108: 192-198.

8) Sathian K, Greenspan AI, Wolf SL: Doing it with mirrors: a case study of a novel approach to neurorehabilitation. Neurorehabil Neural Repair, 2000, 14: 73-76.

9) Stevens JA, Stoykov ME: Using motor imagery in the rehabilitation of hemiparesis. Arch Phys Med Rehabil, 2003, 84: 1090-1092.

10) Stevens JA, Stoykov ME: Simulation of bilateral movement training through mirror reflection: a case report demonstrating an occupational therapy technique for hemiparesis. Top Stroke Rehabil, 2004, 11: 59-66.

11) Maki A, Yamashita $Y$, Ito $Y$, et al.: Spatial and temporal analysis of human motor activity using noninvasive NIR topography. Med Phys, 1995, 22: 1997-2005.

12) Kato $H$, Izumiyama $M$, Koizumi $H$, et al.: Nearinfrared spectroscopic topography as a tool to monitor motor reorganization after hemiparetic stroke: a comparison with functional MRI. Stroke, 2002, 33: 2032-2036.
13) Okamoto $M$, Dan H, Sakamoto K, et al.: Threedimensional probabilistic anatomical cranio-cerebral correlation via the international 10-20 system oriented for transcranial functional brain mapping. Neuroimage, 2004, 21: 99-111.

14) Tsuzuki D, Jurcak V, Singh AK, et al.: Virtual spatial registration of stand-alone fNIRS data to MNI space. Neuroimage, 2007, 34: 1506-1518.

15) Shimada S, Hiraki K: Infant's brain responses to live and televised action. Neuroimage, 2006, 32: 930-939.

16) Takeda K, Gomi Y, Imai I, et al.: Shift of motor activation areas during recovery from hemiparesis after cerebral infarction: A longitudinal study with near-infrared spectroscopy. Neurosci Res, 2007, 59: 136-144.

17) Kim SG, Ashe J, Hendrich K, et al.: Functional magnetic resonance imaging of motor cortex: hemispheric asymmetry and handedness. Science, 1993, 261: 615-617.

18) Garry MI, Loftus A, Summers JJ: Mirror, mirror on the wall: viewing a mirror reflection of unilateral hand movements facilitates ipsilateral M1 excitability. Exp Brain Res, 2005, 163: 118-122.

19) Nudo RJ, Wise BM, SiFuentes F, et al:: Neural substrates for the effects of rehabilitative training on motor recovery after ischemic infarct. Science, 1996, 272: 1791-1794.

20) Levy CE, Nichols DS, Schmalbrock PM, et al.: Functional MRI evidence of cortical reorganization in upper-limb stroke hemiplegia treated with constraintinduced movement therapy. Am J Phys Med Rehabil, 2001, 80: 4-12.

21) Kimberley TJ, Khandekar G, Skraba LL, et al.: Neural substrates for motor imagery in severe hemiparesis. Neurorehabil Neural Repair, 2006, 20: 268-277.

22) Calautti $C$, Naccarato $M$, Jones PS, et al.: The relationship between motor deficit and hemisphere activation balance after stroke: A $3 \mathrm{~T}$ fMRI study. Neuroimage, 2007, 34: 322-331.

23) Chollet F, DiPiero V, Wise RJ, et al.: The functional anatomy of motor recovery after stroke in humans: a study with positron emission tomography. Ann Neurol, 1991, 29: 63-71.

24) Marshall RS, Perera GM, Lazar RM, et al.: Evolution of cortical activation during recovery from corticospinal tract infarction. Stroke, 2000, 31: 656661.

25) Small SL, Hlustik P, Noll DC, et al.: Cerebellar hemispheric activation ipsilateral to the paretic hand correlates with functional recovery after stroke. Brain, 2002, 125: 1544-1557. 